\section{Severe Acute Allergic Response to Intraorbital Polymethylmethacrylate Implant}

\section{Dear Editor,}

Enucleation is the definitive treatment for unilateral extensive intraocular retinoblastoma qualifying as ICRB (International Classification of Retinoblastoma) group E retinoblastoma [1]. There are various orbital implant materials for volume replacement after the removal of the eye. These can be non-porous like polymethylmethacrylate (PMMA) or porous like hydroxyapatite.

The overall reported complications of intraorbital acrylic implants are very low (3.65\%), among them pyogenic granuloma and implants exposure are the frequent ones and had been seen as early postoperative complications [2]. The first ophthalmic allergic reaction to residual monomer of the acrylic resin was reported in 1982 [3]. The reports of allergies to N, N-dimethyl-para-toluidine, in dentistry and orthopedics are also relatively frequent as it is used in certain types of bone cement.

We report a rare case of allergic reaction to PMMA implant leading to intense chemosis which was successfully managed by steroids with retention of the implant. A 4-year-old-male child presented with extensive (group E) intraocular retinoblastoma. He underwent enucleation using the myo-conjunctival technique with a non-porous PMMA intraorbital implant.

Postoperatively, within the first 24 hours, there was the expulsion of the conformer accompanied by underlying conjunctival chemosis. Histopathology report of the enucleated eye was suggestive of intraocular retinoblastoma. The tumor cells were confined within the retinal layers arranged in sheets. The anterior segment structures as well as extraocular muscles and resection end of the optic nerve were free of tumor cells. Keeping the possibility of any

Received: November 30, 2020 Final revision: April 9, 2021

Accepted: April 19, 2021 primary or secondary infections, the patient was started on intravenous and topical antibiotics. There was painless increase in chemosis over the next week with mucopurulent discharge (Fig. 1A). There was no exposure of the implant. The differential blood counts continued to be within normal limits and the patient was afebrile. It was a type IV hypersensitivity reaction supported by the delayed presentation. The debulking of the chemosis was done by aspirating the fluid which was found to be serous. The microbial evaluation of the same was unremarkable.

The patient was given oral steroids $(1 \mathrm{mg} / \mathrm{kg}$ body weight) and the conjunctival chemosis subsided in 2 weeks. After 2 weeks there was an attempt to taper the steroids which showed recurrence of the chemosis. So, the patient was put on oral steroids for another next 4 weeks and then tapered off along with topical steroid ointment. After 3 months of postenucleation, the patient was off steroids and given ocular prosthesis which showed reasonable motility (Fig. 1B).

In the index case, the persistence and the recurrence of the inflammatory symptoms should raise suspicion for a type IV hypersensitivity reaction which takes longer time to initiate than those triggered by antibodies. The risk of hypersensitivity is considered to be depending upon multiple factors including an individual's age, gender, occupation, and a positive history of implant material.

The results of the patch test and history of allergic reactions are regarded as a significant clue to identify the patients who would have hypersensitivity reactions [3]. In view of the non-availability of the allergen kit and patient's response to steroid, the patch test was not performed in the index case. In the present case also there was a definitive
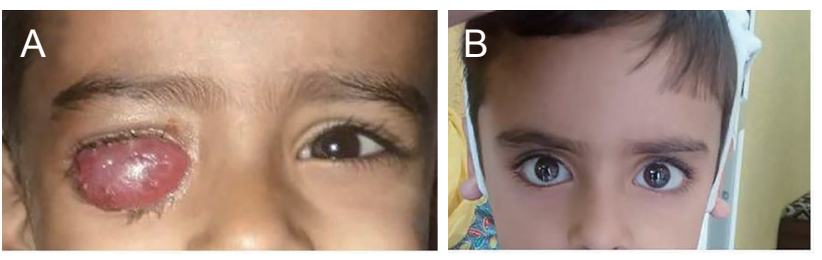

Fig. 1. (A) Clinical photograph of right eye showing extensive conjunctival chemosis with mucopurulent discharge. (B) Same eye showing complete resolution of conjunctival chemosis and well-placed ocular prosthesis. Informed consent for publication of the clinical images was obtained from the parent of the patient. 
history of allergic conjunctivitis. The preoperative and intraoperative steroid coverage could have curtailed the probability of postoperative acute allergic response.

Topical steroids, mast cell stabilizers, and non-steroidal anti-inflammatory drugs have been recommended for acute allergic conjunctivitis secondary to PMMA prosthesis [4]. Change of prosthetic material or revision of the surgery was found to be the definitive treatment in some cases [5]. The index case started showing response to the trial of steroids so the removal of the prosthesis was deferred.

To conclude, postoperative intense conjunctival hyperemia and chemosis in a postenucleated socket of a retinoblastoma patient poses a diagnostic and therapeutic challenge and PMMA implant could show an inflammatory reaction. In such an eventuality, after ruling out any infective pathology, these cases can be managed successfully with prolonged immunosuppression. Discussion of hypersensitivity reactions and their sequelae should be a routine part of preoperative informed consent for ocular implant procedure.

\section{Sonia Phulke, Navya Naveen Kalra}

Department of Ophthalmology, Government Medical College and Hospital, Chandigarh, India

\section{Uma Handa}

Department of Pathology, Government Medical College and Hospital, Chandigarh, India

\section{Subina Narang}

Department of Ophthalmology, Government Medical College and Hospital, Chandigarh, India

E-mail: subina@gmch.gov.in

\section{Conflict of Interest}

No potential conflict of interest relevant to this article was reported.

\section{References}

1. Shields CL, Shields JA. Basic understanding of current classification and management of retinoblastoma. Curr Opin Ophthalmol 2006;17:228-34.

2. Christmas NJ, Gordon CD, Murray TG, et al. Intraorbital implants after enucleation and their complications: a 10year review. Arch Ophthalmol 1998;116:1199-203.

3. Koch KR, Trester W, Muller-Uri N, et al. Ocular prosthetics: fitting, daily use and complications. Ophthalmologe 2016;113:133-42.

4. Patel V, Allen D, Morley AM, Frcophth RM. Features and management of an acute allergic response to acrylic ocular prostheses. Orbit 2009;28:339-41.

5. Jongman HP, Marinkovic M, Notting I, et al. Donor sclerawrapped acrylic orbital implants following enucleation: experience in 179 patients in the Netherlands. Acta Ophthalmol 2016;94:253-6. 